\title{
The epidemic of pre-diabetes: the medicine and the politics
}

During the editing of the table in this Analysis article (BMJ 2014;349:g4485, doi:10.1136/bmj.g4485), the last row and some of the symbols for arterial disease in the impaired glucose tolerance row were inadvertently deleted. The predictive value for arterial disease should also be $+/$ - not + in the rows for expanded impaired fasting glucose and expanded borderline
$\mathrm{HbA}_{1 \mathrm{c}}$. The table has been reproduced in its correct format below.

Cite this as: BMJ 2014;349:94683

๑ BMJ Publishing Group Ltd 2014 


\section{Table}

Table 1| Evidence on value of various definitions of sub-diabetes

Diabetes

Arterial disease

Retinal disease

\begin{tabular}{|c|c|c|c|c|c|c|c|}
\hline & \multicolumn{3}{|c|}{ Diabetes } & \multicolumn{3}{|c|}{ Arterial disease } & \multirow{2}{*}{$\begin{array}{c}\text { Retinal disease } \\
\text { Predicts }\end{array}$} \\
\hline & Predicts & $\begin{array}{c}\text { Effect of lifestyle } \\
\text { interventions }\end{array}$ & Effect of drugs & Predicts & $\begin{array}{c}\text { Effect of } \\
\text { lifestyle } \\
\text { interventions }\end{array}$ & $\begin{array}{l}\text { Effect of } \\
\text { drugs }\end{array}$ & \\
\hline \multirow{2}{*}{$\begin{array}{l}\text { Impaired glucose } \\
\text { tolerance }(7.8-11.1 \\
\mathrm{mmol} / \mathrm{L})^{*}\end{array}$} & & +++ (delays) & +++ (disguises) & & & & \\
\hline & +++ & + (prevents) & + (prevents) & +++ & + & $?$ & + \\
\hline $\begin{array}{l}\text { Impaired fasting glucose } \\
(6.1-6.9 \mathrm{mmol} / \mathrm{L})\end{array}$ & ++ & $?$ & $(+) \dagger$ & + & $?$ & $?$ & $?$ \\
\hline $\begin{array}{l}\text { Expanded impaired fasting } \\
\text { glucose }(5.6-6.9 \mathrm{mmol} / \mathrm{L})\end{array}$ & + & $?$ & $?$ & $+/-$ & $?$ & $?$ & $?$ \\
\hline $\begin{array}{l}\text { Borderline } \mathrm{HbA}_{\urcorner_{1 \mathrm{c}}} \\
(6.0-6.4 \%)\end{array}$ & ++ & $?$ & $?$ & + & $?$ & $?$ & $?$ \\
\hline $\begin{array}{l}\text { Expanded borderline } \mathrm{HbA}_{1 \mathrm{c}} \\
(5.7-6.4 \%)\end{array}$ & + & $?$ & $?$ & $+/-$ & $?$ & $?$ & $?$ \\
\hline Pre-diabetes $\ddagger$ & ++ & $?$ & $?$ & $+/-$ & $?$ & $?$ & $?$ \\
\hline
\end{tabular}

*Two hours after $75 \mathrm{~g}$ glucose load.

† The DREAM Study included $14 \%$ of subjects with impaired fasting glucose in whom rosiglitazone showed comparable effects to those with impaired glucose tolerance at the end of the intervention, ${ }^{37}$ although this group was not reported separately after drug washout. ${ }^{45}$

${ }^{\ddagger}$ Impaired glucose tolerance (7.8-11.1 mmol/L) or impaired fasting glucose 5.6-6.9 mmol/L or expanded borderline $\mathrm{HbA}_{1 \mathrm{c}}(5.7-6.4 \%)$. 